\title{
IMPACTO DA DISTRIBUIÇÃO DE NÚMERO DESIGUAL DE PROGÊNIES POR REPRODUTOR NA AVALIAÇÃO GENÉTICA DE ANIMAIS, EM AMBIENTES COM PRESENÇA DE HETEROGENEIDADE DE VARIÂNCIA AMBIENTAL
}

\author{
IMPACT OF THE UNEVEN DISTRIBUTION OF THE NUMBER OF \\ PROGENIES BY SIRES IN THE GENETIC EVALUATION OF ANIMALS \\ IN ENVIRONMENTS WITH PRESENCE OF HETEROGENEITY OF \\ ENVIRONMENTAL VARIANCE
}

\author{
Claudio Vieira de Araujo ${ }^{1^{*}}$ \\ Lutero de Andrade Oliveira² \\ Simone Inoe Araujo ${ }^{1}$ \\ Delvan Alves da Silva ${ }^{1}$ \\ Alessandra Alves da Silva ${ }^{1}$ \\ ${ }^{1}$ Universidade Federal de Mato Grosso, Sinop, MT, Brasil. \\ ${ }^{2}$ Universidade Federal do Pará, Belém, PA, Brasil. \\ *Autor para correspondência - araujocv@bol.com.br
}

\section{Resumo}

Foi simulado um teste de progênie com o objetivo de avaliar o efeito da heterogeneidade de variância ambiental sobre a avaliação genética de reprodutores, em situações de número desigual de progênie por reprodutor entre rebanhos. A situação do número desigual de progênie por reprodutor foi representada eliminando-se, de forma aleatória, o número de progênies por reprodutor em ambientes de baixa e alta variabilidade ambiental. Nas situações que envolveram perda de informação no ambiente de menor variabilidade ambiental, houve maiores superestimações na estimação da variância residual, ocasionando menores estimativas de herdabilidade. Por outro lado, quando se diminuiu a quantidade de informação proveniente de rebanhos de alta variabilidade ambiental, houve maior superestimação da variância genética aditiva e menor superestimação da variância ambiental. Embora tenham ocorrido alterações na magnitude dos valores genéticos para os reprodutores, as correlações de Spearman e Pearson entre os valores genéticos dos mesmos, em todas as situaçãoes, foram maiores do que 0,90 . O número desigual de progênie por reprodutor na presença de heterogeneidade de variância ambiental provoca superestimação das estimativas de componentes de variância e valores absolutos de predição de valores genéticos dos reprodutores, sem, contudo, alterar a classificação dos mesmos.

Palavras chave: avaliação genética; parâmetros genéticos; seleção.

\begin{abstract}
A progeny test representing the milk yield was simulated to evaluate the effect of heterogeneity of environmental variance on genetic evaluation of sires in situations of unequal number of progeny per sire between herds, eliminating, at random, daughters per sires in environments of low and high environmental variability. In all situations involving loss of information in the environment of lower environmental variability, there was larger overestimation of the residual variance component, resulting
\end{abstract}


in lower estimates of heritability. On the other hand, the decrease in the amount of information of herds from high environmental variability led to greater overestimation of additive genetic variance and lower overestimation of environmental variance. Although there were alterations in breeding values regarding sires, Spearman and Pearson correlations between breeding values, in all situations, were higher than 0.90 . The uneven number of progeny per sire in the presence of heterogeneity of environmental variance led to the overestimation of variance components and changes in absolute breeding values of the sires, without, however, changing the classification values.

Keywords: genetic evaluation; genetic parameters; selection.

Recebido em: 23 novembro 2011

Aceito em: 04 outubro 2016

\section{Introdução}

A heterogeneidade de variâncias pode ser uma forma primária da interação genótipo ambiente e, geralmente, os modelos mistos empregados na avaliação genética de gado de leite assumem variâncias genéticas e residuais homogêneas entre ambientes. A homogeneidade de variâncias não é um requerimento nas análises genéticas, desde que as estimativas de variâncias heterogêneas estejam disponíveis ${ }^{(1)}$.

Se a heterogeneidade de variâncias for ignorada quando existir, a produção de filhas de determinado reprodutor será ponderada na proporção dos desvios-padrão dos rebanhos nos quais elas foram criadas. O resultado é que as produções das filhas em rebanhos mais variáveis influenciarão mais a avaliação de reprodutores do que as produções das filhas oriundas de rebanhos menos variáveis ${ }^{(2)}$.

Um dos principais problemas dos componentes de variâncias heterogêneos na avaliação genética é que animais acima da média podem ser superavaliados nos rebanhos com maior variação e, como resultado, maior proporção de animais provenientes desses rebanhos serão selecionados. A existência de variâncias heterogêneas foi observada por Oliveira et al. ${ }^{(3)}$ nos grupos genéticos participantes da formação da raça Canchim e por Urioste et al. ${ }^{(4)}$ na raça Holandesa.

Marion et al. ${ }^{(5)}$ verificaram relação positiva e crescente dos componentes de variância com o nível de produção de leite dos rebanhos avaliados; no entanto, os coeficientes de herdabilidade não apresentaram o mesmo comportamento, ocorrendo estimativas maiores no nível de baixa produção. A variância genética foi proporcionalmente maior no nível baixo e menor no nível médio de produção, ou seja, com o aumento do nível de produção do rebanho ocorreu uma compressão da variância genética pela variância de ambiente, induzindo a menores coeficientes de herdabilidade no nível médio e a maiores no nível baixo de produção.

Estratificando rebanhos pela produção de leite, em rebanhos da raça Holandesa, Weber et al.(6) observaram menores estimativas de herdabilidade da produção de leite para os níveis baixos e médios, sugerindo que, nestes níveis, a resposta esperada pela seleção poderia ser menos eficiente.

Com informações de lactações em bubalinos, Araújo et al. ${ }^{(7)}$ verificaram médias e componentes de variância maiores para o nível de alta produção, porém com estimativas de herdabilidade menores e correlação genética entre os níveis de 0,58 , caracterizando a presença de heterogeneidade de variância entre os rebanhos.

O objetivo com este estudo foi avaliar, por meio de simulação de dados, o efeito da distribuição de progênies de reprodutores em ambientes com presença de heterogeneidade de variâncias residual e seu impacto na avaliação genética de reprodutores. 


\section{Material e Métodos}

A simulação de dados para estrutura de modelos mistos utilizou a decomposição de Cholesky das matrizes de covariâncias dos efeitos aleatórios do modelo. Assim, em uma situação geral, se $\mathrm{K}$ representa uma matriz de covariância qualquer, sendo $K=U T^{1 / 2} \cdot T^{1 / 2} U^{\prime}$, em que $\mathbf{U T}^{1 / 2}$ é a decomposição de Cholesky da matriz $\mathbf{K}$, e fazendo a transformação $y=\mu+U T^{1 / 2} \cdot Z$, sendo $\mathbf{Z}$ uma matriz de variáveis aleatórias normais padronizadas, então $E(Y)=\mu$ e $V(Y)=0+U T^{1 / 2} I T^{1 / 2} U^{\prime}=K$, em que I é uma matriz identidade. Assim, y possui distribuição normal com média e variância $\mathbf{K}$.

Admitindo que seja criado um conjunto de dados de $\mathrm{N}$ indivíduos com cada indivíduo contendo registro de proles em i níveis de produção, o modelo linear misto é definido como: $y=X \beta+Z a+\varepsilon$, em que y é um vetor de observações dos indivíduos; $\beta$ é um vetor de parâmetros de efeitos fixos; $\mathbf{X}$ é uma matriz de delineamento de efeitos fixos; a é um vetor aleatório de valores representando o efeito genético aditivo; $\mathbf{Z}$ é uma matriz de incidência do efeito genético aditivo; o vetor $\varepsilon$ é um vetor de efeito aleatório de ambiente temporário.

Assumindo que os vetores $\mathbf{y}$, a e $\varepsilon$ apresentam distribuição normal, a esperança do vetor aleatório a é $E(a)=0$ e a variância é $V(a)=A \otimes G_{0}=G$, em que $\mathbf{G}_{0}$ é uma matriz de covariâncias do efeito genético aditivo e $\mathbf{A}$ é a matriz que indica o grau de parentesco entre os indivíduos, de dimensão igual ao número total de indivíduos $(\mathrm{N})$.

Por último, $\varepsilon$ possui média $\mathbf{E}(\boldsymbol{\varepsilon})=\mathbf{0}$ e variância $V(\varepsilon)=I \cdot \sigma_{e}^{2}=R$, em que $\delta^{2}$ é a variância do efeito de ambiente temporário e I uma matriz identidade de dimensão igual ao número de indivíduos com observações (n). Consequentemente, a esperança e a variância do vetor de dados observados y são $E(Y)=X \beta$ e $V(Y)=Z G Z^{\prime}+R$, respectivamente. Ao vetor $\beta$ estão associados os efeitos fixos relacionados ao nível de produção para todos os indivíduos com informação. Se $U T_{a}^{1 / 2}$ é a decomposição de Cholesky das matrizes de covariâncias do efeito genético aditivo e $\mathbf{A}$ é uma matriz de numeradores de coeficiente de parentesco entre os indivíduos, em que $A T_{a}{ }^{1 / 2}$ é a decomposição de Cholesky dessa matriz de parentesco, então o vetor $\mathbf{Y}$ (fenótipos) contendo as "i" níveis de produção é definido como: $Y=\mu+A T_{a}{ }^{1 / 2} Z a .+U T_{a}{ }^{1 / 2}+\boldsymbol{e}$, ou seja, $\mathbf{Y}=$ constante geral + valor genético aditivo + valor de ambiente temporário. Assim seria criado um conjunto de $\boldsymbol{i}$ níveis de produção com distribuição normal, em que cada efeito aleatório possui a sua estrutura própria de covariância, e estes mesmos efeitos aleatórios seriam independentes entre si.

Foram simuladas estruturas de dados em modelos mistos representando a produção de leite para o teste de 100 reprodutores, sendo cada reprodutor acasalado com 10 matrizes (total de 1000 matrizes), originando em cada combinação de acasalamento duas proles, totalizando 2000 proles (vinte proles por reprodutor). De cada combinação de reprodutor e matriz, dez proles apresentaram seu fenótipo expresso no ambiente de baixa produção e, a outra metade, no ambiente de alta produção.

Inicialmente, simulou-se a estrutura de dados com variâncias genéticas aditiva e ambientais homogêneas entre os ambientes, caracterizando o primeiro cenário. Em seguida, obtiveram-se as estimativas de componentes de variância e predições de valores genéticos por meio do aplicativo MTDFREML ${ }^{(8)}$. As estimativas de componentes de variâncias e de herdabilidade obtidas foram bem próximas dos valores simulados e as correlações de Pearson e de Spearman entre valores genéticos preditos e simulados foram próximas de um.

Em um segundo cenário, simulou-se nova estrutura de dados em que a variância residual no estrato 2 apresentou o dobro $\left(2133,00 \mathrm{~kg}^{2}\right)$ da variância do estrato $1\left(1067,04 \mathrm{~kg}^{2}\right)$.

Posteriormente, criaram-se três situações de número desigual de proles por reprodutor. Na primeira 
situação (desbalanceamento 1), 50\% das proles de metade dos pais foram eliminadas aleatoriamente no ambiente de baixa variabilidade ambiental (situação 1).

Em uma segunda situação (desbalanceamento 2), foram eliminadas aleatoriamente $50 \%$ das proles de todos os reprodutores, no ambiente de baixa variabilidade ambiental, caracterizando a segunda situação.

Na terceira situação, (desbalanceamento 3) eliminaram-se aleatoriamente $50 \%$ das proles de todos os reprodutores no ambiente de alta variabilidade ambiental, caracterizando a situação 3 .

De posse das estimativas de componentes de variância, herdabilidade e predição de valores genéticos dos reprodutores, obtidas pelo aplicativo MTDFREML, compararam-se as situações com dados balanceados e dados desbalanceados.

Os valores de componentes de variância utilizados neste estudo foram baseados em Araújo et al. ${ }^{(7)}$. Para simulação dos dados, foi utilizado o programa $\mathrm{SAS}^{(9)}$, utilizando-se o PROC INBREED para o cálculo da matriz de parentesco integrado no mesmo algoritmo ao modulo IML, sobre o qual foi realizada toda a simulação de dados anteriormente descrita.

As análises dos dados em ambos os cenários e em todas as estruturas de dados na obtenção dos componentes de variância, bem como dos valores genéticos preditos para os reprodutores, seguiram o modelo $y=X \beta+Z a+e$, em que y é um vetor $\mathrm{n} x 1$, de $\mathrm{n}$ observações de produção; $\mathrm{X}$ é uma matriz $\mathrm{n} \times \mathrm{f}$, de incidência de $\mathrm{f}$ níveis de produção; $\beta$ é um vetor $\mathrm{f} \times 1$, de efeitos fixos referentes às classes níveis e produção; $\mathrm{Z}$ é uma matriz $\mathrm{n} \times \mathrm{N}$, de incidência dos valores genéticos; $a$ é um vetor $\mathrm{N}$ x 1 , de valores genéticos dos animais e $e$ é um vetor de resíduos da mesma dimensão de y, em que N é o número de indivíduos; $\mathbf{n}$ é o número total de observações e f é o número de classes $\mathbf{d}$ e efeitos fixos.

As pressuposições utilizadas acerca da distribuição dos vetores $\mathbf{y}, \mathbf{a}, \mathbf{p}$ e e são descritas como

$\left[\begin{array}{l}y \\ a \\ e\end{array}\right] \sim\left\{\left[\begin{array}{c}x \beta \\ \emptyset \\ \emptyset\end{array}\right],\left[\begin{array}{ccc}Z G Z^{\prime}+R & Z G & R \\ Z G^{\prime} & G & \emptyset \\ R & \emptyset & R\end{array}\right]\right\}$

em que $G=A \mathrm{o}_{a}^{2}$, e $R=I_{n} \sigma_{\varepsilon}^{2}$, sendo $A$ a matriz de numerador do coeficiente de parentesco entre os indivíduos, de ordem igual ao número de indivíduos $(\mathrm{N})$; e ${ }^{\sigma_{\varepsilon}^{2}}$ $\mathrm{e}^{\sigma_{a}^{2}}$ são os componentes de variância genética aditiva e ambiental para produção de leite; $\mathrm{I}_{\mathrm{n}}$ é a m matriz identidade, de ordem $n$.

\section{Resultados e Discussão}

Observa-se na Tabela 1 que a situação de número desigual de proles por reprodutor nos estratos provocou superestimação dos componentes de variância. Nas situações que envolveram os desbalanceamentos 1 e 2, houve maiores superestimações da variância residual, ocasionando menores estimativas de herdabilidade. Já na situação de desbalanceamento 3, houve maior superestimação da variância genética aditiva.

Na Figura 1, é exibido o percentual de superestimação dos componentes de variância genético aditivo e residual em relação à condição em que todos os reprodutores apresentam o mesmo número de proles por estrato. Ao se diminuir a contribuição da informação fenotípica proveniente dos rebanhos de menor variabilidade ambiental, o incremento da variância ambiental foi substancialmente maior. Por outro lado, quando se diminuiu a quantidade de informação proveniente de rebanhos de alta variabilidade ambiental, produziu-se maior superestimação da variância genética aditiva e menor superestimação da variância ambiental. 
Tabela 1. Estimativa de componentes de variância genético aditivo, residual e herdabilidade $\left(h^{2}\right)$ em condição de número igual e desigual de proles por reprodutor, em presença de heterogeneidade de variância residual

\begin{tabular}{lcccc}
\hline & & \multicolumn{3}{c}{ Desbalanceamento } \\
\cline { 3 - 5 } & Balanceado & $\mathbf{1}$ & $\mathbf{2}$ & $\mathbf{3}$ \\
\hline Aditivo & 747105,21 & 992107,31 & 1007704,01 & 1221511,03 \\
& & & & \\
Residual & 1099345,61 & 2812025,00 & 3147178,91 & 1854072,88 \\
& & & & \\
\hline $\mathrm{h}^{2}$ & 0,40 & 0,26 & 0,24 & 0,39 \\
\hline
\end{tabular}

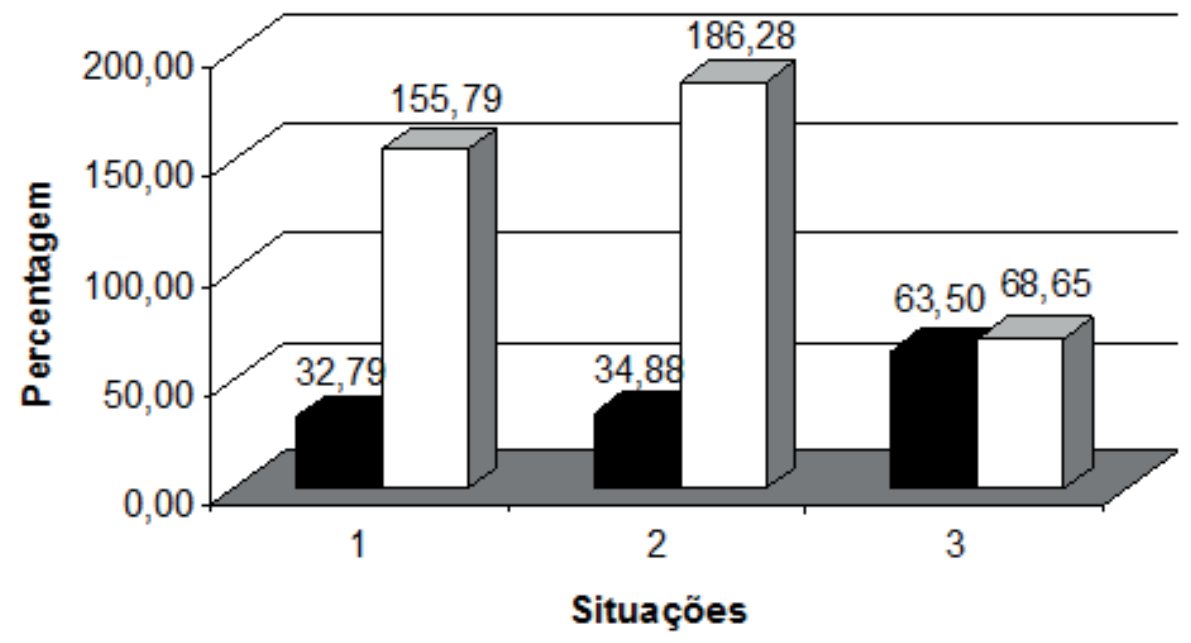

Aditiva $\square$ Residual

Figura 1. Representação gráfica do percentual de superestimação dos componentes de variância aditiva e residual, na presença de heterogeneidade de variância, com situações envolvendo diferentes números de proles por reprodutor.

Verifica-se na Tabela 2 que o número desigual de proles por reprodutor no estrato de baixa variabilidade superestima a predição do valor genético dos reprodutores e diminui a amplitude entre as predições, havendo menor discriminação entre as predições de valores genéticos dos reprodutores, quando comparada à situação em que o número desigual de proles ocorreu no estrato de alta variabilidade.

Carneiro et al. ${ }^{(10)}$ estudaram os efeitos de heterogeneidade sobre a avaliação genética de bovinos, simulando várias estruturas de dados com heterogeneidade para diferentes parâmetros, com e sem conexidade genética entre rebanhos. Os autores verificaram que para as estruturas de dados com médias genéticas similares e heterogeneidade para outros parâmetros, as correlações entre valores genéticos foram superiores a 70\%, além disso, alta conexidade genética dos dados melhorou a predição dos valores genéticos de touros.

A origem da heterogeneidade de variância é proveniente de diferenças ambientais, o valor genético dos reprodutores é ponderado pelo desempenho de suas proles em cada estrato. Quando se diminui a contribuição do estrato de baixa variabilidade, o valor genético é predito com base no 
desempenho de animais com maiores produções, superestimando as predições dos valores genéticos (desbalanceamento 1 e 2). Quando a contribuição do estrato de alta variabilidade diminui na predição do valor genético dos reprodutores, também se diminui a superestimação das predições dos valores genéticos (desbalanceamento 3).

Tabela 2. Estatística descritiva para os valores genético preditos dos reprodutores em diferentes situações envolvendo diferentes números de proles por reprodutor na presença de heterogeneidade de variância residual

\begin{tabular}{lccc}
\hline Condição & Mediana & Mínimo & Máximo \\
\hline Balanceado & $-17,03$ & $-1578,00$ & 1616,00 \\
Desbalanceado 1 & 67,82 & $-1452,00$ & 1700,00 \\
Desbalanceado 2 & 80,45 & $-1406,00$ & 1695,00 \\
Desbalanceado 3 & 49,47 & $-1807,00$ & 2024,00 \\
\hline
\end{tabular}

Porém, mesmo havendo alteração na magnitude dos valores genéticos preditos para os reprodutores, a heterogeneidade de variância não alterou a classificação entre os reprodutores (Tabela 3), todas as correlações de ordem foram próximas à unidade. Observou-se que na situação de desbalanceamento 3, apesar de seguir o mesmo comportamento das outras situações, a oscilação dos valores em relação ao balanceamento foi bem maior.

Verificando-se a magnitude da heterogeneidade da variância da produção de leite dentro de rebanho e o efeito de ajustamento para a mesma sobre os valores genéticos em animais da raça Holandesa, Teixeira et al. ${ }^{(11)}$ constataram que foi baixo o efeito do ajustamento para heterogeneidade da variância sobre os valores genéticos dos touros.

Em estudo de simulação de dados, Carneiro et al. ${ }^{(10)}$ verificaram que a existência de conexidade genética entre rebanhos resultou em aumento nas correlações de ordem entre valores genéticos verdadeiros e preditos. Quando os touros tinham progênies em todos os rebanhos, as correlações de ordem para touros foram superiores a $90 \%$. Situação contrária foi observada para o caso em que a conexidade genética foi baixa.

Tabela 3. Correlação de Pearson (acima da diagonal) e de Spearman (abaixo da diagonal) entre os valores genético preditos dos reprodutores $(n=100)$ em diferentes situações envolvendo diferentes números de proles por reprodutor, na presença de heterogeneidade de variância residual

\begin{tabular}{lcccc}
\hline & Balanceado & $\begin{array}{c}\text { Desbalanceado } \\
\mathbf{1}\end{array}$ & $\begin{array}{c}\text { Desbalanceado } \\
\mathbf{2}\end{array}$ & $\begin{array}{c}\text { Desbalanceado } \\
\mathbf{3}\end{array}$ \\
\hline Balanceado & 1,00 & 0,96 & 0,96 & 0,90 \\
Desbalanceado 1 & 0,89 & 1,00 & 0,99 & 0,95 \\
Desbalanceado 2 & 0,87 & 0,98 & 1,00 & 0,95 \\
Desbalanceado 3 & 0,91 & 0,95 & 0,93 & 1,00 \\
\hline
\end{tabular}

Carneiro Júnior et al. ${ }^{(12)}$, por meio de dados simulados, com a finalidade de verificar a influência da heterogeneidade de variância residual na predição dos valores genéticos, utilizando inferência bayesiana, em dois tamanhos de população (pequeno $(n=300)$ e grande $(n=4.800))$, observaram que heterogeneidade residual prejudica a predição dos valores genéticos e que a utilização da informação a priori informativa, apesar de diminuir os quadrados médios dos erros, não resulta em melhor 
classificação dos indivíduos.

Carvalheiro et al. ${ }^{(13)}$, também por meio de simulação de dados, avaliando os efeitos da heterogeneidade de variância residual entre grupos de contemporâneos na avaliação genética de bovinos de corte, verificaram que esses efeitos estão associados à pressão de seleção e aos níveis de heterogeneidade, sendo que, se correções não forem feitas, rebanhos que praticam seleção intensa e que apresentam níveis acentuados de heterogeneidade de variância residual podem ter seus animais classificados de maneira incorreta e, consequentemente, menos resposta à seleção.

Torres et al. ${ }^{(2)}$ observaram altas correlações entre os valores genéticos dos reprodutores para a produção de leite em diferentes classes de desvios-padrão, desconsiderando a heterogeneidade de variâncias. Entretanto, os reprodutores apresentaram maiores valores genéticos em rebanhos das classes de alto desvio-padrão, similarmente ao verificado neste estudo. Ainda, os autores concluíram que, na avaliação genética, é importante considerar a variabilidade entre rebanhos, pois, sob seleção, as classes mais variáveis contribuíram com a maior parte dos animais, e a avaliação genética do animal poderia ser função não apenas do seu potencial genético, mas também do ambiente no qual suas progênies expressaram a característica.

Campêlo etal. ${ }^{(14)}$ também constataram que, apesar das variâncias genética e residual, das herdabilidades, das médias e dos desvios-padrão dos valores genéticos dos reprodutores terem aumentado com o aumento do desvio padrão fenotípico da classe, a heterogeneidade de variâncias não causou mudança na ordem de classificação dos reprodutores da raça Tabapuã.

Por outro lado, Araújo et al. ${ }^{(15)}$ encontraram evidências da presença de heterogeneidade de variância sobre a produção de leite em animais da raça Pardo Suíça. Verificaram que reprodutores com proles em rebanhos mais variáveis estariam sendo melhores classificados na avaliação genética, quando se desconsidera a heterogeneidade de variância.

Mudanças na classificação dos animais, sob diferentes modelos que consideravam ou não a heterogeneidade de variância em características de crescimento e de carcaça de animais de diferentes grupos genéticos, foram observadas por Nuñez-Dominguez et al. ${ }^{(16)}$ e Crews e Franke ${ }^{(17)}$.

A distribuição desigual de progênie por reprodutor entre diferentes ambientes, na presença de heterogeneidade residual, provoca maior superestimação no componente de variância ambiental com perda de informação no ambiente menos variável e maior superestimação da variância genética aditiva com perda de informação no ambiente de maior variabilidade ambiental.

A perda de informação no ambiente mais variável provoca maior amplitude das predições dos valores genéticos dos reprodutores, sem alterar a classificação do ordenamento dos mesmos.

\section{Conclusões}

A distribuição desigual de progênie por reprodutor entre diferentes ambientes associada à presença de heterogeneidade residual provoca maior superestimação na variância ambiental e variância genética aditiva, acarretando maior amplitude para as predições dos valores genéticos dos reprodutores, sem, no entanto, alterar a classificação do ordenamento dos mesmos.

\section{Referências}

1. Henderson CR. Application of linear models in animal breeding. Guelph, Canadá: Ithaca: University of Guelph; 1984. 462 p. 
2. Torres RA, Bergmann JAG, Costa CN, Pereira CS, Valente J, Penna VM, et al. Heterogeneidade de variância e avaliação genética de bovinos da raça Holandesa no Brasil. Rev Bras Zootec [periódico na Internet]. 2000;29(4):1050-9. Disponível em: http://dx.doi.org/10.15-90/S1516-35982000000400015.

3. Oliveira CAL, Martins EN, Freitas AR, Alencar MM. Heterogeneidade de Variâncias nos Grupos Genéticos Formadores da Raça Canchim. Rev Bras Zootec [periódico na Internet]. 2001;30(4):1212-9. Disponível em: http://dx.doi.org/10.1590/S1516-35982001000500012.

4. Urioste JI, Rekaya R, Gianola D, Fikse WF, Weigel KA. Model comparison for genetic evaluation of milk yield in Uruguayan Holsteins. Livest Prod Sci [periódico na Internet]. 2003;84(1):63-73. Disponível em: http://dx.doi.org/ 10.1016/S0301-6226(03)000514.

5. Marion AE, Roberto P, Rorato N, Ferreira GB, Magda D, Everling F, et al. Estudo da Heterogeneidade das Variâncias para as Características Produtivas de Rebanhos da Raça Holandesa no Rio Grande do Sul. Rev Bras Zootec [periódico na Internet]. 2001;30(6S):1995-2001. Disponível em: http://dx.doi.org/ 10.1590/S151635982001000800006.

6. Weber T, Rorato PRN, Ferreira GBB, Boligon AA, Gheller DG, Guterres LFW. Coeficientes de herdabilidade e correlações genéticas para as produções de leite e de gordura, em diferentes níveis de produção, para raça Holandesa no estado do Rio Grande do Sul. Rev Bras Zootec [periódico na Internet]. 2005;34(2):514-9. Disponível em: http://dx.doi.org/10.1590/S1516-35982005000500011.

7. Araújo CV, Cardoso, A. M. C. Ramos AA, Araújo SI, Marques JRF, Tomazini API, Chaves LC. Heterogeneidade de variâncias e parâmetros genéticos para produção de leite em bubalinos da raça Murrah, mediante inferência Bayesiana. Rev Bras Saúde e Produção Anim. 2008;9(3):416-25.

8. Boldman KG, Kriese LA, Van Vleck LD., Van TasselL, CP; Kachman SD. Manual for use of MTDFREML; a set of programs to obtain estimates of variance and covariance [DRAFT]. Lincoln: Department of Agriculture Research Service; 1995. p. 120.

9. SAS Institute Inc. SAS/STAT User’s Guide, Version 7-1. Cary, NC: SAS Institute Inc; 1999.

10. Carneiro PAS, Torres RDA, Lopes PS, Euclydes RF, Carneiro PLS, Silva FF. Heterogeneidade e avaliação genética em bovinos, estudo utilizando dados simulados. Acta Sci Anim Sci [periódico na Internet]. 2008;30(1):113-9. Disponível em: http://dx.doi.org/-10.4025/actascianimsci.v30i1.3621.

11. Teixeira NM, Freitas AF, Ferreira WJ, Durães MC, Barra RB. Ajustamento para Heterogeneidade de Variância da Produção de Leite de Vacas da Raça Holandesa no Estado de Minas Gerais. Rev Bras Zootec [periódico na Internet]. 2002; 31(S1):369-75. Disponível em: http://dx.doi.org/ 10.1590/S1516-35982002000200011.

12. Carneiro Junior JM, Assis GML, Euclydes RF, Torres RDA, Lopes PS. Estimação de componentes de variância utilizando-se inferência Bayesiana e freqüentista em dados simulados sob heterogeneidade de variâncias. Rev Bras Zootec [periódico na Internet]. 2007;36(5):1539-48. Disponível em: http://dx.doi. org/10.1590/S1516-35982007000700012.

13. Carvalheiro R, Fries LA, Schenkel FS, Albuquerque LG. Efeitos da Heterogeneidade de Variância Residual entre Grupos de Contemporâneos na Avaliação Genética de Bovinos de Corte. Rev Bras Zootec [periódico na Internet]. 2002;31(4):1680-8. Disponível em: http://dx.doi.org/10.1590/S1516-35982002000700010.

14. Campelo JE de G, LopesPS, Torres RDA, SilvaLOC, Euclydes RF, Araújo CV, etal. Estudo deheterogeneidade de variâncias na avaliação genética de bovinos de corte da raça Nelore. Rev Bras Zootec [periódico na Internet]. 2011;40(9):1902-8. Disponível em: http://dx.doi.org/10.1590/S1516-35982011000900009.

15. Araújo CV, Resende GSA, Araújo SI, Rennó FP, Tomazini API, Marques JRF. Interação genótipo x ambiete para produção de leite na raça Pardo Suíço, utilizando-se inferência Bayesiana. Acta Sci - Anim Sci [periódico 
na Internet]. 2009;31(2):205-11. Disponível em: http://dx.doi.org/10.4025/actascianimsci.v31i2.5197.

16. Núnez-Dominguez R, Van Vleck LD, Cundiff L V. Prediction of genetic values of sires for growth traits of crossbred cattle using a multivariate animal model with heterogeneous variances. J Anim Sci [periódico na Internet]. 1995;73(10):2940-50. Disponível em: http://dx.doi.org/10.2527/1995.73102940x.

17. Crews DH, Franke DE. Heterogeneity of Variances for Carcass Traits by Percentage Brahman Inheritance. J Anim Sci [periódico na Internet]. 1998;76(7):1803-9. Disponível em: http://dx.doi.org/ doi:10.2527/1998.7671803x. 